\title{
Addressing the Unfolding Children's Mental Health Crisis
}

\author{
Eric A. Storch ${ }^{1} \cdot$ Erica Ding ${ }^{1}$ \\ Published online: 16 January 2022 \\ (c) The Author(s), under exclusive licence to Springer Science+Business Media, LLC, part of Springer Nature 2021
}

2021 is a year of transitions. As our understanding of COVID-19 and the implementation of COVID-19 vaccines and boosters continue to evolve, society at large attempts to regain semblances of normalcy. Airline travel resumes to allow for families to gather during holidays, roads are once again filled with workers rushing to and from their offices, and schools for our children convert from virtual back to inperson. Yet, despite all our efforts, we cannot turn the clock back to the pre-pandemic days. As of December 2021, the United States (U.S.) has lost almost eight hundred thousand people to the virus, and the world over five million [1,2]. Many more people continue to suffer from disabilities associated with COVID treatment and recovery, or even "long COVID", ongoing symptoms and conditions the medical world is only starting to quantify. The tremendous loss of human life is only compounded by loss of jobs, financial security, and trust in public health and governmental officials [3]. With so many stressors, it is no wonder that reports of depressive and anxiety symptoms and substance use have increased since the pandemic began [4]. The pandemic's impact on mental health is widespread, and as we wrestle with having to live with COVID-19 and its numerous evolutions, enduring. This is especially true for the mental health toll on our children, the youngest of whom had never known the days prior to March 2020. In this editorial, we outline the mental health crisis affecting our vulnerable children and adolescents, how the pandemic persists in affecting their mental health, and how we have and will continue to address this crisis.

The ongoing mental health challenge facing children and adolescents is not new. Historically, suicide ranks amongst the top five causes of death for adolescents, sometimes second only to deaths caused by unintentional injuries such as motor vehicle accidents [5, 6]. Even prior to the

Eric A. Storch

Eric.storch@bcm.edu

1 Department of Psychiatry and Behavioral Sciences, Baylor College of Medicine, 1977 Butler Blvd, Mailpoint 350, Houston, TX 77030, USA pandemic, the U.S. national prevalence of children with at least one mental health disorder was $16.5 \%$, which equated to about 7.7 million children, with approximately half of them untreated [7]. Once COVID-19 lockdown began, there was further decline in utilization of pediatric mental health services, likely attributable to many of these essential services being offered through schools [8]. At the same time, the proportion of mental health-related visits to emergency departments (ED) increased, despite overall decreases in total number of ED visits during the initial phase of the lockdown [9]. The same study demonstrated that while the number of more routine ED visits, such as for asthma and otitis media, decreased, ED visits for psychosocial stressors rose to comprise $69 \%$ of total visits. Stress related to the disruption of daily life and routines, illness anxiety, and social isolation were all cited as factors that triggered or exacerbated mental illness. While 12 to 17 year-olds accounted for the highest proportion of mental health-related visits, young children were in no way exempt from the effects of stress and anxiety. In fact, 3 to 5 year-olds demonstrated higher levels of social and emotional difficulties compared to previous years. In these children's formative years, their participation in preschool programs decreased by almost $25 \%$, and many parents were simply not satisfied with the quality of remotely attending programs [10].

These are just a small sample of the statistics that demonstrate the mental health challenges facing our children in the U.S. Globally, one in seven children have been affected by lockdowns in some fashion, and amongst adolescents and young adults aged 15 to 24 years, one in five cited feelings of depression or loss of interests [11]. Worldwide, children are suffering; not just from the virus, but the economic, social, and educational impacts from lockdown. As these measures ease, however, transitioning back to normal life poses its own unique challenges.

What normalcy means post-pandemic, and whether or not there even is such a thing as "after" the pandemic, varies. As of December 2021, almost $60 \%$ of U.S. adults are fully vaccinated, but fears of COVID-19 variants and surges continue to impact masking policies and restrictions. 
Despite ongoing uncertainties, many are returning to in-person work, and importantly, children have largely returned to in-person schooling since Fall of 2021. The American Academy of Pediatrics (AAP) endorsed in-person learning for all age groups, with masking and full vaccination for groups eligible for the vaccine, most recently expanded to 5 to 11 year-olds [12]. Remote learning proved to exacerbate existing educational disparities, especially obvious amongst children with disabilities, children from families in poverty, and racial minorities. Yet, reopening and transitioning can be another source of stress for many children. According to the Center for Disease Control (CDC), for infants and young children, many were born right before or in the midst of the pandemic, and they and their caregivers might have had limited exposure to childhood socialization and early childhood programs [13]. Upon reopening, children had to adjust to new routines and meet new authority figures, not to mention learn to get along with their peers in new environments. Adolescents, too, faced similar struggles, in addition to education concerns and test anxieties. Of course, these stressors would be even more overwhelming for children with existing developmental, behavioral, and emotional issues. The need for adequate mental health resources for children cannot be overstated: from pre-pandemic to now, approaching two years into the pandemic, and looking to the future, equitable access to adequate and appropriate pediatric mental health care must be a priority for all.

Thankfully, due to the hard work of many advocates, governments and various agencies have increasingly recognized the need to address this mental health crisis affecting children. In Texas, for example, there has been unprecedented efforts within the Texas Children's Mental Health Care Consortium to expand child mental health services through various programs including telepsychiatry into schools, pediatrician consultation services, and workforce expansion. As the pandemic continues, however, mental health problems are certain to continue, requiring continued and extensive efforts to prevent another public health emergency. We hope that this editorial brings continued attention to the challenges that affect children and families as innovative efforts will be necessary to address the persisting mental health crisis.
2. WHO (2021) WHO coronavirus (COVID-19) dashboard. https:// covid19.who.int/. Accessed 30 Nov 2021

3. Han Q, Zheng B, Cristea M, Agostini M, Bélanger JJ, Gützkow B, Kreienkamp J, PsyCorona Collaboration, Leander NP (2021) Trust in government regarding COVID-19 and its associations with preventive health behaviour and prosocial behaviour during the pandemic: a cross-sectional and longitudinal study. Psychol Med. https://doi.org/10.1017/S0033291721001306

4. Panchal N, Kamal R, Cox C, Garfield R, Chidambaram P (2021) Mental health and substance use considerations among children during the COVID-19 pandemic. https://www.kff.org/coronaviruscovid-19/issue-brief/mental-health-and-substance-use-considerat ions-among-children-during-the-covid-19-pandemic/. Accessed 30 Nov 2021

5. Miniño AM (2010) Mortality among teenagers aged $12-19$ years: United states, 1999-2006 (NCHS data brief No. 37). National Center for Health Statistics: CDC, Hyattsville, MD

6. Van Orman A, Jarosz B (2016) Suicide replaces homicide as second-leading cause of death among U.S. teenagers. https://www. prb.org/resources/suicide-replaces-homicide-as-second-leadingcause-of-death-among-u-s-teenagers/. Accessed 30 Nov 2021

7. Whitney DG, Peterson MD (2019) US national and state-level prevalence of mental health disorders and disparities of mental health care use in children. JAMA Pediatr 173(4):389-391

8. Panchal N, Kamal R, Cox C, Garfield R (2021) The implications of COVID-19 for mental health and substance use. https://www. kff.org/coronavirus-covid-19/issue-brief/the-implications-ofcovid-19-for-mental-health-and-substance-use/. Accessed $30 \mathrm{Nov}$ 2021

9. Leeb RT, Bitsko RH, Radhakrishnan L, Martinez P, Njai R, Holland KM (2020) Mental health-related emergency department visits among children aged $<18$ years during the COVID-19 pandemic-United States, January 1-October 17, 2020 (Morbidity and Mortality Weekly Report No. 69). CDC

10. Barnett SW, Jung K (2021) Seven impacts of the pandemic on young children and their parents: initial findings from NIEER's December 2020 preschool learning activities survey. National Institute for Early Education Research, New Brunswick

11. UNICEF (2021) Impact of COVID-19 on poor mental health in children and young people 'tip of the iceberg'. UNICEF, New York

12. Korioth T (2021) AAP urges in-person learning, masking in updated guidance on safe schools. American Academy of Pediatrics. https://publications.aap.org/aapnews/news/17241?autologinc heck=redirecteded

13. CDC (2021) Helping children transition back to school. https:// www.cdc.gov/childrensmentalhealth/features/COVID-19-helpingchildren-transition-back-to-school.html. Accessed 30 Nov 2021

Publisher's Note Springer Nature remains neutral with regard to jurisdictional claims in published maps and institutional affiliations.

\section{References}

1. CDC (2021) CDC COVID data tracker. https://covid.cdc.gov/ covid-data-tracker/. Accessed 30 Nov 2021 